\title{
Tubes, tables and traps: great apes solve two functionally equivalent trap tasks but show no evidence of transfer across tasks
}

\author{
Gema Martin-Ordas · Josep Call • \\ Fernando Colmenares
}

Received: 15 October 2007 / Revised: 30 November 2007 / Accepted: 13 December 2007 / Published online: 9 January 2008

(C) Springer-Verlag 2007

\begin{abstract}
Previous studies on tool using have shown that presenting subjects with certain modifications in the experimental setup can substantially improve their performance. However, procedural modifications (e.g. trap table task) may not only remove task constraints but also simplify the problem conceptually. The goal of this study was to design a variation of the trap-table that was functionally equivalent to the trap-tube task. In this new task, the subjects had to decide where to insert the tool and in which direction the reward should be pushed. We also administered a trap-tube task that allowed animals to push or rake the reward with the tool to compare the subjects' performance on both tasks. We used a larger sample of subjects than in previous studies and from all the four species of great apes (Gorilla gorilla, Pan troglodytes, Pan paniscus, and Pongo pygmaeus). The results showed that apes performed better in the trap-platform task than in the trap-tube task. Subjects solved the tube task faster than in previous studies and they also preferred to rake in rather than to push the reward out. There was no correlation in the level of performance between both tasks, and no indication of interspecies differences. These data are consistent with the idea that apes may possess some specific causal knowledge of traps but may lack the ability to establish analogical relations between functional equivalent tasks.
\end{abstract}

G. Martin-Ordas $(\bowtie) \cdot$ J. Call

Max Planck Institute for Evolutionary Anthropology,

Deutscher Platz 6, 04103 Leipzig, Germany

e-mail: ordas@eva.mpg.de

G. Martin-Ordas · F. Colmenares

Departamento de Psicobiología de la Facultad de Psicología de la Universidad Complutense de Madrid, Madrid, Spain
Keywords Tool-use $\cdot$ Action constraints Primates · Causal knowledge $\cdot$ Analogy

\section{Introduction}

Tool using is an indirect means of achieving a goal by causally relating objects to one another (Tomasello and Call 1997). Although a variety of animals are capable of using tools to solve problems (e.g., Beck 1980; Natale et al. 1988; Tomasello and Call 1997; Chappell and Kacelnik 2002; Tebbich and Bshary 2004; Helme et al. 2006), their degree of understanding of the problem features (tools, obstacles and rewards) and the causal relationships between those features is still an unresolved question. One task that has received substantial research attention is the trap-tube task (Visalberghi and Limongelli 1994). Capuchin monkeys were presented with a plexiglass tube from which they had to extract a reward without pushing it inside a trap situated in the centre of the tube. Visalberghi and Limongelli (1994) found that after 120 trials only one of four subjects solved the task by pushing the reward away from the trap. However, this subject persisted in her strategy during the control condition, in which the trap was inverted 180 degrees, rendering it non-functional. The authors concluded that this subject learnt to insert the tool into the side farthest away from the reward without understanding the causal relations between the trap and the reward.

Limongelli et al. (1995) presented the trap-tube task to five chimpanzees. However, this task differed from the one presented to the capuchins in the control condition. Limongelli et al. (1995) changed the ineffective trap from the centre to a functional trap placed closer to one end of the tube opening. In some cases the trap was located on the right side of the tube, while in others the opposite arrangement 
was used. The reward was positioned in the centre of the tube. Two chimpanzees solved the problem and, interestingly, they also avoided the trap in the control condition. The variations made on the tube controlled for the distantbased procedural rule by omitting the inverted the trap control condition. However, this does not rule out the possibility that the chimpanzees could have solved the task by applying the procedural rule "push the reward away from the trap". Povinelli (2000) replicated this experiment including the inverted trap control condition; but his results did not differ much from those obtained with the original task. Only one chimpanzee avoided the trap during the experimental condition; however, this subject also behaved as if the trap was functional in the control condition. Therefore, Povinelli (2000) suggested that the chimpanzee did not understand the causal nature of the task, but used the procedural rule. Silva et al. (2005) have proposed that if there is no cost in avoiding a non-functional trap, it is misleading to assume that an animal that does this has understood nothing about causality. In fact, they found that adult humans do not avoid the ineffective trap. Recently, Seed et al. (2006) tested eight rooks (Corvus frugileus) with a modified version of the trap tube, the two-trap tube task. Subjects were presented with two tubes containing two traps along each tube: a functional and a non-functional trap. In one of these tubes, the reward had to be dragged over the top of the non-functional trap, whereas in the other tube, the reward was supposed to fall through the functional trap and recuperate from below. Therefore, by incorporating a non-functional 'trap' they circumvented the problem of using the inverted trap as a control task. Their results showed that seven rooks rapidly mastered the problem and transferred the solution to a visually and conceptually similar task; however, six of them were unable to transfer this knowledge to conceptually similar but visually different tasks. On the other hand, Tebbich et al. (2007) presented the original trap-tube task and two different two-trap tube transfer tasks to seven rooks. They found that three rooks solved the trap-tube problem; however, none of these rooks passed the transfer tasks (Tebbich et al. 2007). The authors concluded that, even though rooks "are capable of sophisticated physical cognition", they probably do not understand the unobservable causal properties of the trap problems.

It has been argued that part of the difficulty of the traptube may not be due to a lack of understanding of its features, but to the intervention of other variables that could mask their performance. The diameter of the tube and the tool force the animals to push the reward out. This strategy might increase the difficulty of the problem, at least for non-human primates, since pushing the reward away increases the distance between them and the food (Guillaume and Meyerson 1930; Köhler 1925). In line with this observation, Tebbich and Bshary (2004) presented six woodpecker finches (Cactospiza pallida) with a modified trap tube problem. In this task, birds were allowed to either push away or rake in the reward out of the tube, the latter being the action that birds preferred to use. Nevertheless, only one subject solved the task after 100 trials. Additionally, this subject inserted the tool on each side multiple times in successful trials, suggesting that it was not anticipating the effect that its actions would have on the reward in relation to the trap. What this result indicates is that the subject did not understand the causal relation between the tool and the trap. Mulcahy and Call (2006) also presented a modified trap-tube task to great apes in which the subjects could choose between raking or pushing the reward out. This study revealed two interesting results. First, although only three of the ten subjects tested were above chance, they solved the task much faster than any other animals tested before. More important, their performance in the inverted trap condition ruled out the possibility that subjects were simply using the position of the trap as a cue to decide where to insert the tool. Second, animals preferred to rake in rather than to push the reward out of the tube. Mulcahy and Call (2006) suggested that forcing the subjects to get the reward by pushing it out might have caused the poor performance reported in previous trap-tube experiments.

Povinelli (2000) also attempted to simplify the trap-tube problem by administering an equivalent trap task with a different setup. Six chimpanzees were faced with a table divided into two sections. A hole, functioning as a trap, was cut in one of these sections. A rectangle of the same dimensions, placed in a position similar to that of the hole, was painted in the other section. Subjects were given a choice of pulling one of two rakes, which had been placed on the table, until they got the reward. The results indicated that overall, chimpanzees showed no preference for pulling from the rake behind the fake trap. Povinelli (2000) concluded that chimpanzees had little understanding of the trap, or the effect it had on the reward. Subsequent studies carried out with capuchins and gibbons using a similar paradigm also concluded that subjects did not have a total comprehension of the elements of the problem, but that they might have learnt certain associative rules to solve the problem (Fujita et al. 2003; Cunningham et al. 2006). On the other hand, Santos et al. (2006) presented tamarins and vervet monkeys with a battery of raking tasks in which the properties of the tools and the properties of the substrate were altered. The authors found that only vervet monkeys selected the appropriate tool accordingly to its properties and avoided pulling food over a trap, in a performance comparable to that of chimpanzees on such tasks (Povinelli 2000). These results suggest that sensitivity to the relation between the functional aspects of a tool and a substrate are present in non-tool-using primates. 
Girndt et al. (in press) have suggested that the traditional trap-table setup used by Povinelli (2000) possesses some constraints that might have favoured the poor performance of the apes. Girndt et al. (in press) tested the four species of great apes on the original trap-table setup, but also on one in which they varied the type of trap (two tables vs. one table), the position (pre-positioned vs. non-positioned) and the number (one vs. two) of presented tools, the type of tool (rake vs. stick) and the reinforcement regime (non-differentially vs. differentially reinforcement). Their results demonstrated that the four species of great apes avoided the trap (also in the first trial) when they were allowed to insert the tool in one of the two sections of the table but not when the tools were pre-positioned on the table. Therefore, the crucial variable in explaining the discrepancy between their different results and those found by Povinelli (2000) appears to be the position of the tool on the platform. The authors concluded that apes might have possessed causal knowledge about traps but that pre-positioning the tools on the table may have concealed this knowledge.

Although associative explanations cannot be ruled out in some of the studies carried out with non-human primates, recent studies on great apes have provided some evidence that is inconsistent with a purely associative account (Mulcahy and Call 2006; Girndt et al. in press). Some studies have reported successful first trial performance, while others have shown that certain modifications of the experimental procedure can substantially improve the subjects' performance revealing capabilities not previously reported. However, procedural modifications may also simplify the problem conceptually. In particular, the trap-table is not comparable to the trap-tube because there were two rewards in the trap-table and subjects just had to select the alternative without a trap in front. Therefore, the first goal of this study was to design a variation of the trap-table (platform task) that was more similar to the original traptube task since subjects had to decide where to insert the tool and then displace the reward towards one side. We tested representatives of all the great ape species excluding humans. The subjects faced an inverted U-shaped platform with a reward placed on its centre and a trap to one side of the reward. The solution consisted of using the tool to displace the reward towards the side of the platform without the trap to bring it within reach down the arm of the U-shaped platform.

The second goal of this study was to compare the great apes' performance on the platform task to a modified version of the trap-tube task in which both raking and pushing the reward out were possible (e.g. Mulcahy and Call 2006). This comparison allowed us to assess both the relative difficulty of each task and to correlate their scores to investigate whether subjects were capable of transferring the solution across tasks. We predicted that subjects would perform better in the trap-platform than the trap-tube because subjects had performed better in a task in which the trap was presented on a platform compared to a tube (chimpanzees: Povinelli 2000; all great apes: Mulcahy and Call 2006; Girndt et al. in press). We also expect the subjects to perform better in the trap-platform because they had performed better in a modified version of the table task (Girndt et al. in press) than in a modified version of the tube task (Mulcahy and Call 2006). Based on a previous study (Mulcahy and Call 2006), we also predicted that subjects would prefer to rake rather than to push the reward out of the tube. Moreover, since subjects faced no restrictions on the actions that they could use, that is either pushing or raking the reward were possible, we predicted that the subjects would perform better in the current tube task than in previous studies (e.g. Limongelli et al., 1995; Povinelli 2000). Finally, we expected a positive correlation between the performances in both tasks because they were conceived as functionally equivalent.

\section{Materials and methods}

\section{Subjects}

Six orangutans (Pongo pygmaeus), six chimpanzees (Pan troglodytes), five bonobos (Pan paniscus) and three gorillas (Gorilla gorilla) housed at the Wolfgang Köhler Primate Research Center in the Leipzig Zoo participated in this experiment (see Table 1). There were 15 females and 5 males. All apes were mother-reared except six individuals (see Table 1). All subjects except Kila had participated in a variety of cognitive tests and some of them included tasks involving tools and traps (see Table 1). All subjects lived in social groups of various sizes, with access to indoor and outdoor areas that were furnished with natural vegetation, climbing structures, and enrichment devices to foster extractive foraging activity during the day that included the use of tools. Subjects were individually tested (except one of the orangutans) in their indoor cages and were not deprived either of food or water.

\section{Apparatus}

There were two apparatuses: the trap platform and the trap tube. The trap platform was a U-shaped platform, which consisted of a $70-\mathrm{cm}$ wide $\times 31 \mathrm{~cm}$ long plastic platform surrounded on three sides by a $2-\mathrm{cm}$ border that prevented the reward from falling off the platform. The front part of the platform was cut to create a trap $(8 \mathrm{~cm}$ wide $\times 15 \mathrm{~cm}$ long) and to prevent subjects from getting the reward from its starting position (see Fig. 1). There was a border on part of the front side of the platform to prevent the reward from falling into empty space. 
Table 1 Name, gender, age, rearing history, and previous experience of the subjects in tool using in trap tasks

\begin{tabular}{|c|c|c|c|c|}
\hline Subject & Gender & $\begin{array}{l}\text { Age } \\
\text { (months) }\end{array}$ & $\begin{array}{l}\text { Rearing } \\
\text { history }\end{array}$ & $\begin{array}{l}\text { Previous } \\
\text { experience } \\
\text { in trap tasks }\end{array}$ \\
\hline
\end{tabular}

\begin{tabular}{lllll}
\hline Chimpanzee & & & & \\
Fraukje & F & 360 & Nursery raised & 2,3 \\
Jahaga & F & 162 & Mother raised & 3 \\
Fifi & F & 156 & Mother raised & $1,2,3$ \\
Trudi & F & 156 & Mother raised & 3 \\
Sandra & F & 156 & Mother raised & 2 \\
Pia & F & 78 & Mother raised & 3 \\
Bonobo & & & & \\
Joey & M & 282 & Nursery raised & $1^{\mathrm{F}}$ \\
Limbuko & $\mathrm{M}$ & 126 & Nursery raised & 3 \\
Kuno & $\mathrm{M}$ & 114 & Nursery raised & 3 \\
Ulindi & $\mathrm{F}$ & 150 & Mother raised & $1^{\mathrm{F}}$ \\
Yasa & $\mathrm{F}$ & 120 & Unknown & 3 \\
Orangután & & & & \\
Bimbo & $\mathrm{M}$ & 306 & Nursery raised & $1^{\mathrm{F}, 3}$ \\
Dunja & $\mathrm{F}$ & 396 & Nursery raised & 3 \\
Pini & $\mathrm{F}$ & 216 & Mother raised & $1^{\mathrm{F}}, 3$ \\
Dokana & $\mathrm{F}$ & 198 & Mother raised & $1^{\mathrm{F}, 3}$ \\
Padana & $\mathrm{F}$ & 102 & Mother raised & 3 \\
Kila & $\mathrm{F}$ & 72 & Mother raised & - \\
Gorilla & & & & \\
Gorgo & $\mathrm{M}$ & 300 & Nursery raised & 3 \\
Ndiki & $\mathrm{F}$ & 342 & Mother raised & 3 \\
Viringika & $\mathrm{F}$ & 132 & Mother raised & $1^{\mathrm{F}, 3}$ \\
\hline I & & & & \\
\hline
\end{tabular}

1 trap-tube task, ( $I^{F}$ subjects failed the task, Mulcahy and Call 2006); 2 tube task (Seed et al. unpublished data); 3 trap table (Girndt et al. in press)

The trap tube followed the design by Mulcahy and Call (2006), which was a modified version of the one used by Visalberghi and Limongelli (1994). For the current study we used two tubes. Each consisted of a $40 \mathrm{~cm}$ long $\times 5 \mathrm{~cm}$ diameter plexiglass tube with an off-centre opaque trap ( $8 \mathrm{~cm}$ wide $\times 15 \mathrm{~cm}$ long) located in its bottom part. A small hole was drilled in both traps, so when the reward dropped into it, the experimenter could take the reward away. One tube had the trap on the right side $(8 \mathrm{~cm}$ from the right end of the tube) and the other had the trap on the left side ( $8 \mathrm{~cm}$ from the left end of the tube). The distance between the reward and the trap was identical in both tasks: $8 \mathrm{~cm}$. Both tubes had a small hole $(1 \mathrm{~cm}$ diameter $)$ drilled in the middle of their back wall to allow the experimenter to bait them.

The tool consisted of a straight wooden dowel $(0.5 \mathrm{~cm}$ diameter) with a length of $50 \mathrm{~cm}$ in the trap platform condition and $40 \mathrm{~cm}$ in the trap tube condition.

\section{Procedure}

There were two phases: pretest and test. In the pretest, subjects were allowed to retrieve the reward from an apparatus (platform and tube) without traps to see if they were proficient at using tools. If they retrieved the reward with smooth movements, they moved to the next phase. Subjects received one 10-trial session except the gorilla Gorgo that received two sessions to ensure that he could manipulate the tool proficiently in the trap tube condition. In the test, subjects received two tasks:

\section{Platform}

The experimenter placed the trap-platform outside the cage against the mesh and deposited half of a grape (or a banana slice for the orang-utan Bimbo) on a predetermined spot in the centre of the platform equidistant to the retrieval areas (Fig. 1). The experimenter gave the tool to the subject through the mesh at a point located just above the reward so that no side bias was induced. To retrieve the reward the
Fig. 1 Experimental setup for the trap-platform (a) and the trap-tube tasks (b)

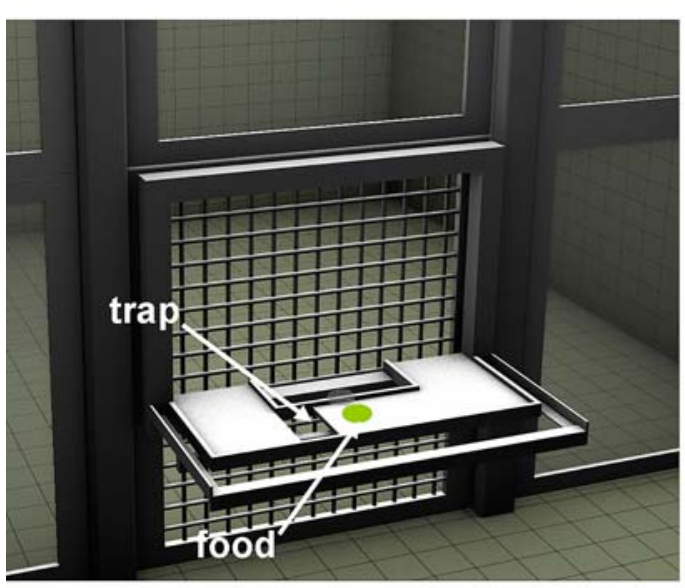

(a) Trap-platform

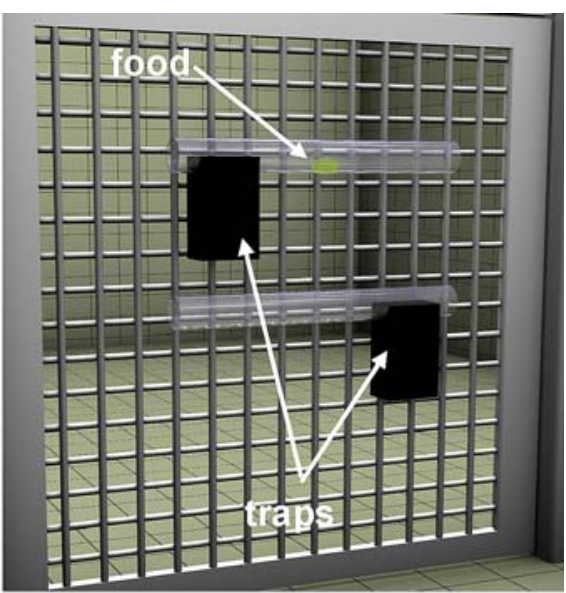

(b) Trap-tube 
subject had to take the tool, insert it through the mesh and move the reward away from the trap.

\section{Tube}

The experimenter placed both trap-tubes inside the cage fastened to the mesh with metal braces. The tubes were located one above the other with their respective traps facing opposite sides (Fig. 1). The location of the trap (left-right) for each tube changed across sessions, but remained the same during a session. The experimenter placed the reward inside one of the tubes through the baiting hole and gave the tool to the subject through the mesh right above the apparatus. In order to get the reward, subjects had to insert the stick through one of the sides of the tube and rake or push the reward away from the trap.

In both tasks, the trial ended after the subject retrieved the reward or when the reward fell into the trap. Subjects received both tasks sequentially, with half of the subjects receiving the platform first and half of the subjects receiving it second. All subjects participated in three 12trial sessions per condition (72 trials in total) except Gorgo that participated in a total of 66 trials. He stopped cooperating after 30 trials with the trap tube. The position of the trap or which tube was baited was counterbalanced across trials within a session so that it appeared the same number of times to the left and to the right of the subject.

Data scoring and analysis

We videotaped all trials. For each trial we scored whether they retrieved the reward. Additionally and only for the trap-tube condition, we scored whether they inserted the tool from one side of the tube only and the technique that they used to displace the reward. We distinguished four techniques:

Rake: displace the reward toward the tool's insertion point.

Push: displace the reward away from the tool's insertion point.

Mixed: insert the tool from one side and pull it out from the other side.

Wiggle: shake the tool once it is inside the tube.

We calculated the percentage of trials in which subjects (1) obtained the reward, (2) used each of the above-mentioned techniques, and (3) inserted the tool from only one end of the tube. We conducted parametric tests whenever the data met the assumptions for its use. Unless it was otherwise indicated, all tests were two-tailed. Those for which we had a priori predictions were one-tailed.

\section{Results}

Success

Figure 2 presents the percentage of correct trials for each condition across sessions. A mixed model ANOVA with condition and session as within-subject factors and species as between-subject factor indicated that subjects performed better in the platform compared to the tube condition $\left(F_{1,16}=3.46, P=0.04\right.$, one-tailed $)$ and improved their performance across sessions $\left(F_{2,16}=5.99, P=0.006\right)$. However, there was no evidence of improvement in the subjects' performance within sessions for the tube condition (first session: $t_{19}=1.16, P=0.26$; second session: tube: $t_{19}=0.25, P=0.81$; third session: $t_{18}=0.59$, $P=0.56)$. Subjects' performance did not change within the first two sessions for the platform condition, although it did improve within the third session (first session: $t_{19}=1.64$, $P=0.12$; second session: $t_{19}=0.27, P=0.79$; third session: $\left.t_{19}=2.65, P=0.016\right)$. No other factors or interactions were significant. A re-analysis with species excluded confirmed these results (condition: $F_{1,16}=5.04, P=0.019$, one-tailed; session: $\left.F_{2,16}=7.58, P=0.004\right)$. Overall, subjects were above chance in all sessions for both conditions $\left(t_{19}>3.05, P<0.01\right.$ in all cases) except the second session of the tube condition $\left(t_{19}=1.77, P=0.092\right)$. However, subjects were not above chance in the first trial of the platform $\left(\chi^{2}=3.20, d f=1, P=0.074,14 / 20\right.$ correct $)$ or tube conditions $\left(\chi^{2}=0.80, d f=1, P=0.37,12 / 20\right.$ correct). There was no evidence that subjects who had previous experience with trap-tube tasks performed better than subjects who did not have that experience $\left(t_{18}=0.35, P=0.37\right.$, one-tailed; see Table 1). In addition, the number of trap tasks to which the subject had been exposed had no significant effect on the scores of the trap-tube task $(r=0.33, P=0.077$, one-tailed) and trap-platform task ( $r=0.27, P=0.12$, one-tailed).

Table 2 presents the subjects' individual performance in each task. Five subjects solved both tasks, seven solved the platform task only, three solved the tube task only and five

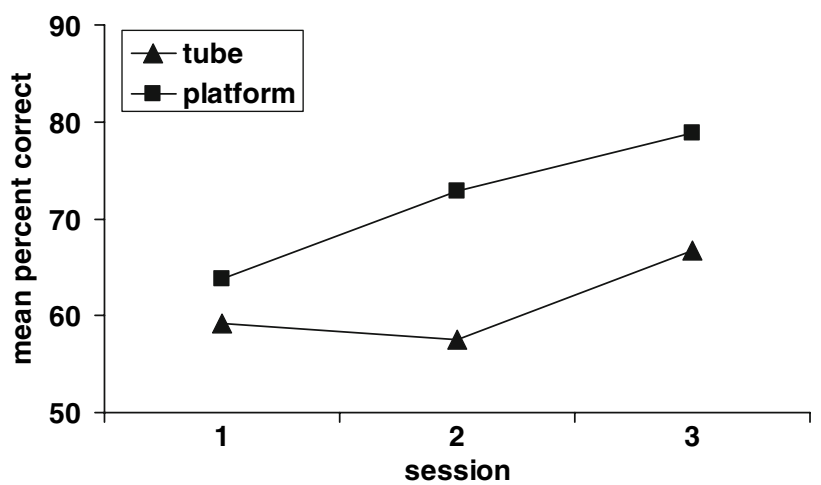

Fig. 2 Percentage of correct trials for each task across sessions 
Table 2 Percentage of correct responses in both tasks, significance levels on binomial tests and session in which subjects are above chance

\begin{tabular}{|c|c|c|c|c|c|c|}
\hline \multirow[t]{3}{*}{ Subject } & \multicolumn{6}{|l|}{ Task } \\
\hline & \multicolumn{3}{|l|}{ Platform } & \multicolumn{3}{|l|}{ Tube } \\
\hline & $\begin{array}{l}\text { Correct } \\
(\%)\end{array}$ & $P$ & Session & $\begin{array}{l}\text { Correct } \\
(\%)\end{array}$ & $P$ & Session \\
\hline \multicolumn{7}{|c|}{ Chimpanzee } \\
\hline Fraukje & 52.77 & 0.868 & - & 52.77 & 0.868 & - \\
\hline Jahaga & 83.33 & $<0.001$ & 2 & 58.33 & 0.405 & - \\
\hline Fifi & 94.44 & $<0.001$ & 1 & 77.77 & $<0.001$ & 3 \\
\hline Trudi & 100 & $<0.001$ & 1 & 58.33 & 0.405 & - \\
\hline Sandra & 47.22 & 0.868 & - & 77.77 & 0.001 & 3 \\
\hline Pia & 69.44 & 0.029 & 3 & 91.66 & $<0.001$ & 1 \\
\hline \multicolumn{7}{|l|}{ Bonobo } \\
\hline Joey & 88.88 & $<0.001$ & 2 & 55.55 & 0.618 & - \\
\hline Limbuko & 97.22 & $<0.001$ & 1 & 47.22 & 0.868 & - \\
\hline Kuno & 75 & 0.004 & 3 & 69.44 & 0.029 & 3 \\
\hline Ulindi & 55.55 & 0.618 & - & 77.77 & 0.001 & 2 \\
\hline Yasa & 63.88 & 0.132 & - & 55.55 & 0.618 & - \\
\hline \multicolumn{7}{|l|}{ Orangutan } \\
\hline Bimbo & 75 & 0.004 & 2 & 55.55 & 0.618 & - \\
\hline Dunja & 75 & 0.004 & 3 & 58.33 & 0.405 & - \\
\hline Pini & 77.77 & 0.001 & 2 & 69.44 & 0.029 & 3 \\
\hline Dokana & 97.22 & $<0.001$ & 1 & 69.44 & 0.029 & 3 \\
\hline Padana & 80.55 & $<0.001$ & 2 & 58.33 & 0.405 & - \\
\hline Kila & 44.44 & 0.618 & - & 27.77 & 0.989 & - \\
\hline \multicolumn{7}{|l|}{ Gorilla } \\
\hline Gorgo & 50 & 1 & - & 50 & 1 & - \\
\hline Ndiki & 55.55 & 0.618 & - & 47.22 & 0.868 & - \\
\hline Viringika & 52.77 & 0.868 & - & 69.44 & 0.029 & 3 \\
\hline
\end{tabular}

solved neither task. There was no relationship in levels of performance between both tasks $(r=0.13, P=0.57$, $n=20$ ), that is, subjects who solved one task did not necessarily solve the other. Table 2 also presents the speed at which subjects reached above chance performance. There were no significant differences between the platform and the tube task (Wilcoxon test: $z=1.85, P=0.065$ ).

Retrieval tube technique

Figure 3 shows the percentage of trials in which subjects used each technique to retrieve the reward from the tube. Subjects significantly preferred to rake than push the reward out (Wilcoxon test: $\mathrm{z}=1.98, P=0.024$, one-tailed). Moreover, subjects also preferred the raking technique compared to the mixed (Wilcoxon test: $z=2.56, P=0.01$ ) and the wiggle techniques (Wilcoxon test $z=3.68$, $P<0.001)$. We also analysed the strategies used during the pretest. Subjects significantly preferred to rake (mean $=$

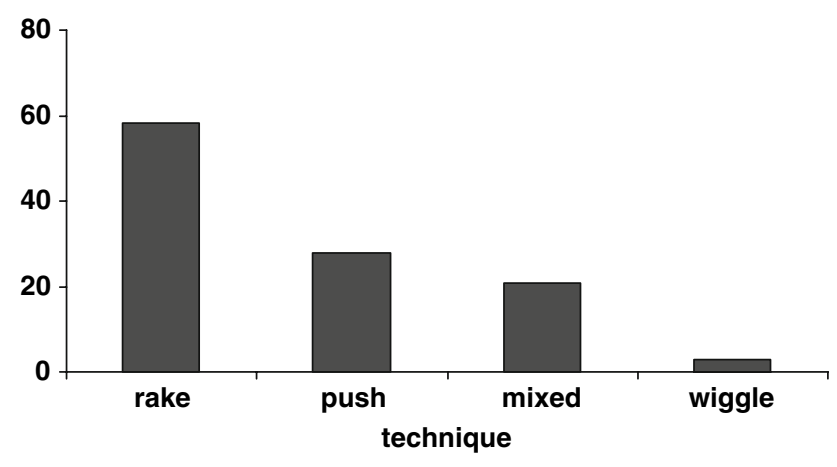

Fig. 3 Percentage of trials in which subjects used each technique to retrieve the reward in the trap-tube task

$64.5 \%, \mathrm{SEM}=8.7)$ than to push the reward out $(\mathrm{z}=2.84$, $P=0.004$, mean $=18 \%, \mathrm{SEM}=5.2$ ) or use one of the combined techniques (mixed: $\mathrm{z}=-.8, P=0.005$, mean $=$ $15 \%, \mathrm{SEM}=5.5 ;$ mixed $z=3.76, P<0.001$, mean $=1.5 \%$, $\mathrm{SEM}=1.5$ ).

Most of the subjects, attempting to retrieve the reward, used single tool insertions. Only in $14.2 \%$ ( $\mathrm{SEM}=2.6$ ) of the trials did subjects insert the tool into both sides of the tube.

\section{Discussion}

Apes performed better in the trap platform task than in the trap tube task. Twelve subjects out of 20 solved the platform problem, whereas only eight were able to solve the trap tube task. Subjects tended to be faster solving the platform problem than the tube problem: four apes solved the platform task within the first 12 trials, whereas only one individual solved the tube task within the first 12 trials. We found no evidence of inter-specific differences. There was no correlation in the level of performance between the two tasks. Our subjects solved the tube task faster (and in greater numbers) than in previous studies and they also preferred to rake in rather than to push the reward out. Previous experience with trap tasks did not influence the subjects' performance. Moreover, in successful trials, subjects normally used single tool insertions, supporting the idea that they could have anticipated the solution of the problem.

The poor performance of the subjects on the trap tasks reported in some of the previous studies (Visalberghi and Limongelli 1994; Povinelli 2000; Fujita et al. 2003) contrasts with the better performance observed in the current study. As predicted, subjects performed better in the platform task than in the tube task and, moreover, they were above chance in the first session (four subjects were above chance individually). One reason that could explain the better performance in the platform task is that, as Povinelli (2000) proposed, this is a less demanding task because individuals do not need to mentally represent the side into which 
they could insert the tool, only the consequence of their actions over the reward. However, in our platform task, subjects had to insert the tool before they used it, yet their group performance was above chance in the first session.

Another possibility is that the trap platform affords the use of actions such as raking a reward for which apes show a great predisposition, clearly greater than pushing the reward away (e.g. Guillaume and Meyerson 1930; Köhler 1925). Indeed, Mulcahy and Call (2006) found that great apes prefer to rake rather than push a reward out of the tube. The current study confirmed this finding. Additionally, the platform setup may offer subjects a more intuitive type of problem because the trap is located between the reward and the subject. In contrast, the trap in the tube setup is located in front of the subject but next to the reward. Therefore, the spatial dispositions of the problem elements (including the subject) paired with the subject's preferences for using certain actions may increase the difficulty of the tube tasks compared to platform/table tasks.

Given the challenge posed by the tube task, it is remarkable that $40 \%$ of the subjects $(8 / 20)$ in the current study were above chance after 36 trials. Only one orangutan out of 22 apes tested in previous studies had succeeded after 36 trials (Limongelli et al. 1995; Povinelli 2000; Mulcahy and Call 2006). What can explain these substantial differences among studies? We found no evidence that the amount of experience with trap tasks had an effect on the performance in the current study. In fact, performing well with the first task in the current study had no effect on the performance in the second one. Mulcahy and Call (2006) suggested that allowing subjects to rake (as opposed to push) the reward out of the tube lifted a task constraint that masked the subjects' knowledge about the task. Note that individuals in the current study also preferred to rake the reward-an action that primates and woodpecker finches spontaneously use to get out-of-reach rewards (Köhler 1925; Guillaume and Meyerson 1930; Tebbich and Bshary 2004).

Although this explanation could account for the differences between the initial studies (Limongelli et al. 1995; Povinelli 2000) and subsequent ones (Mulcahy and Call 2006, the present one), it does not account for the difference between the Mulcahy and Call's (2006) study and the current study because raking was possible in both studies. However, there were other differences between these two studies. First, unlike Mulcahy and Call's setup in which the tube was positioned between two cages, we placed the trap tubes in one cage. Mulcahy and Call (2006) suggested that the strong preference by some subjects for moving to one cage might have confounded the selection of the correct location. By placing both tubes in one cage we might have avoided this problem. Second, the length of the tube and of the tool could have influenced the performance of the subjects. In this study, the tube and the tool were much shorter than in Mulcahy and Call's study (tube 40 vs. $95 \mathrm{~cm}$, respectively; tool: 20 vs. $120 \mathrm{~cm}$, respectively). Providing subjects with a longer tool to be inserted into a longer tube may be disadvantageous to their performance. Individuals might have an inaccurate control over the reward, which increases the likelihood of pushing it into the trap.

Third, presenting two trap tubes simultaneously instead of one may have allowed subjects to learn which particular movements produced the reward in each tube. Note that although the placement of the tubes changed across sessions, it remained the same during each session. Additionally, the double-tube setup may have allowed subjects to directly compare the two trap orientations-something that was not possible with the single-tube setup. An increased contrast between alternatives may have facilitated the task by calling attention to the different trap orientations. Such information would be particularly valuable if subjects tended to associate an action with an object and had difficulty switching actions depending on the orientation of the object.

Although gorillas appeared to perform worse than other apes in the current tasks, we found no statistically significant differences between species. However, our reduced sample size may explain this to some extent. Incidentally, the lack of species differences in the current study suggests that orangutans are not more proficient than other apes in this task, a possibility that Mulcahy and Call (2006) had considered. However, future studies with larger samples of gorillas, bonobos, chimpanzees and orangutans are necessary to confirm the lack of interespecific differences.

We found no relation between the performance in the platform and tube tasks. Perhaps administering subjects a larger number of trials may have revealed a positive relation between tasks. In any case, the lack of association between tasks was unexpected because we considered them as different instantiations of the same problem. The reasons for this outcome remain unclear and we can only speculate at this point. One possibility is that subjects had no causal knowledge about the task but had associated certain actions with success in a particular task without any understanding of the relations between the elements of the problem. However, this explanation does not fit well with some of the recent data on other trap-tube tasks in apes (Girndt et al., in press; Mulcahy and Call 2006; Seed 2007) and corvids (Seed et al. 2006) that may suggest some understanding of the trap properties. Another possibility is that apes possessed some causal knowledge but it could not be expressed because it was tied to particular actions as it has been shown in previous studies (e.g. Girndt et al. in press). For instance, Mulcahy and Call (2006) found that those subjects, who solved the trap tube when pushing and raking were possible, failed when they could only push the reward out of the tube. 
Alternatively, actions per se may not have been the problem but the subjects' causal knowledge may be tied to specific tasks. This means that apes may have difficulty in establishing an analogy between functionally equivalent tasks (Gentner and Markman 1997; Gentner 2002), or they may lack a general understanding of the principles that govern all kinds of traps. For instance, subjects may comprehend the relation between the reward, the stick, and the hole in the tube task, but they do not see that the relation between those elements is equivalent (and governed by similar principles) to the relations between the reward, the stick, and the hole on the platform task. Future research is required to test the validity of these various hypotheses.

In conclusion, subjects solved a trap problem on a platform closer to the original trap-tube task. Additionally, subjects performed better in the current trap-tube task than in the previous studies. We propose that the spatial disposition of the problem as well as the actions required to solve the problem were instrumental in producing these results. These results taken together with other recent studies suggest that nonhuman great apes may know more about the relation between traps, rewards and tools than previously thought. This knowledge, however, may be task specific and may not easily permeate across tasks, perhaps due to a limitation in apes' analogical reasoning abilities. Future studies will be needed to confirm the extent of apes' causal knowledge in various problems and to explore further their analogical capabilities.

Acknowledgments This work was partly supported by grants HA2005-0010 (Acciones Integradas between Spain and Germany) from the Spanish Ministry of Education and Science and CCG06UCM/HUM-1187 from the Universidad Complutense of Madrid and Comunidad Autónoma de Madrid to F. Colmenares. Gema Martín-Ordas was supported by a FPI studentship from the Spanish Ministry of Education and Science. The experiments comply with the current laws of the country in which they were performed.

\section{References}

Beck BB (1980) Animal tool behavior: the use and manufacture of tools by animals. Garland STPM Press, New York

Cunningham CL, Anderson JR, Mootnick AR (2006) Object manipulation to obtain a food reward in hoolock gibbons, Bunopithecus hoolock. Anim Behav 71:621-629
Chappell J, Kacelnik A (2002) Tool selectivity in a non-primate, the New Caledonian Crow (Corvus monoduloides). Anim Cogn 5:71-78

Fujita K, Kuroshima H, Asai S (2003) How do tufted capuchin monkeys (Cebus apella) understand causality involved in tool use? J Exp Psychol Anim B 29:233-242

Gentner D (2002) Analogical reasoning, psychology of Encyclopedia of Cognitive Science. Nature Publishing Group, London

Gentner D, Markman AB (1997) Structure mapping in analogy and similarity. Am Psychol 52:45-56

Girndt A, Meier T, Call J (in press) Task constraints mask great apes' ability to solve the trap table task. J Exp Psychol Anim Behav Proc

Guillaume P, Meyerson I (1930) Recherches sur l'usage de l'instrument chez les singes. I. Le probleme du detour. J Psychol Norm Pathol 27:177-236

Helme A, Call J, Clayton NS, Emery NJ (2006) What do bonobos (Pan paniscus) understand about physical contact? J Comp Psychol 120:294-302

Köhler W (1925) The mentality of apes. Vintage Books, New York

Limongelli L, Boysen ST, Visalberghi E (1995) Comprehension of cause-effect relations in a tool-using task by chimpanzees (Pan troglodytes). J Comp Psychol 109:18-26

Mulcahy NJ, Call J (2006) How great apes perform on a modified traptube task. Anim Cogn 9:193-199

Natale F, Poti P, Spinozzi G (1988) Development of tool use in a macaque and a gorilla. Primates 29:413-416

Povinelli DJ (2000) Folk physics for apes: a chimpanzee's theory of how the mind works. Oxford University Press, Oxford

Santos LR, Pearson HM, Spaepen GM, Tsao F, Hauser MD (2006) Probing the limits of tool competence: experiments with two nontool-using species (Cercopithecus aethiops and Saguinus oedipus). Anim Cogn 9:94-109

Seed AM (2007) Cognition in rooks and chimpanzees: a case of convergent evolution? Unpublished Ph.D. Thesis

Seed AM, Tebbich S, Emery NJ, Clayton NS (2006) Investigating physical cognition in rooks, Corvus frugilegus. Curr Biol 16:697701

Silva FJ, Page DM, Silva KM (2005) Methodological-conceptual problems on the study of chimpanzees' folk physics: how studies with adult humans can help. Learn Behav 33:47-58

Tebbich S, Bshary R (2004) Cognitive abilities related to tool use in the woodpecker finch, Cactospiza pallida. Anim Behav 67:689-697

Tebbich S, Seed AM, Emery NJ, Clayton NS (2007) Non-tool-using rooks (Corvus frigilegus) solve the trap-tube task. Anim Cogn 10:225-231

Tomasello M, Call J (1997) Primate cognition. Oxford University Press, New York

Visalberghi E, Limongelli L (1994) Lack of Comprehension of causeeffect relations in tool-using capuchin monkeys (Cebus apella). J Comp Psychol 108:15-22 